\title{
The overview of the spin physics at RHIC-PHENIX experiment
}

\author{
Yoshinori Fukao for the PHENIX Collaboration \\ Department of Physics, Kyoto University, Kyoto, 606-8502, Japan
}

\begin{abstract}
.
In Spiring 2005, RHIC successfully completed its first long data collection run with polarized proton beams. PHENIX accumulated ten fold larger statistics with higher polarization than the previous spin physics run in 2003. This contribution will introduce the RHIC-PHENIX experiment and present our recent results.
\end{abstract}

Keywords: proton, spin structure, asymmetry

PACS: $13.85 . \mathrm{Ni}, 13.88 .+\mathrm{e}, 21.10 . \mathrm{Hw}, 25.40 . \mathrm{Fq}, 14.20 . \mathrm{Dh}, 13.60 . \mathrm{Hb}$

\section{INTRODUCTION}

One of the major goals of the PHENIX spin program is to experimentally study the origin of the proton spin. Past measurements in polarized deep inelastic scattering (DIS) have led to the so-called "spin crisis": quarks and anti-quarks carry only $\sim 30 \%$ of the nucleon spin[2]. Contributions from gluon spin and orbital angular momentum of partons remain unknown.

In the past we reported the double helicity asymmetry $\left(A_{L L}\right)$ for inclusive $\pi^{0}$ production[3]. The measurement of the $A_{L L}$ is one of the promising approaches to the gluon polarization in the proton $(\Delta g)$. In the 2005 run, RHIC has operated for 3 months for spin physics with center-of-mass energy $(\sqrt{s})$ of $200 \mathrm{GeV}$ and average beam polarization of $47 \%$. PHENIX accumulated an integrated luminosity of $\sim 3.8 \mathrm{pb}^{-1}$ with longitudinal polarization and $\sim 0.1 \mathrm{pb}^{-1}$ with transverse polarization. The greatly improved RHIC performance allows a wide-range of measurements using different probes including pions, electrons, muons, prompt photons and jets as well as the $\pi^{0}$ s.

In addition to the measurement of $\Delta g$, studies in progress include single transversespin asymmetries $\left(A_{N}\right)$, spin transfer to the $\Lambda$ and $\mathrm{J} / \psi$ polarization. Towards the highest energy run at $\sqrt{s}=500 \mathrm{GeV}$ planned in near future, polarized beams were successfully accelerated and brought into collisions at $\sqrt{s}=410 \mathrm{GeV}$ in the 2005 run.

\section{PHENIX DETECTOR}

The PHENIX detector[1] consists of global detectors and four spectrometers. One of the global detectors is a set of beam-beam counters (BBC) for the determination of the vertex position of the collision. The minimum bias trigger (MB) is provided by $\mathrm{BBC}$ and the number of $\mathrm{MB}$ events is used as the relative luminosity for the asymmetry analysis. The other detector is a zero degree calorimeter (ZDC) located at very forward region. The ZDC also plays an important role in the polarized proton run as the local polarimeter, which monitors the direction of the beam polarization by measuring the neutron $A_{N}$ and the longitudinal component was more than $98 \%$ during the 2005 run. 
Two spectrometers out of four are constructed in the central region (central arm) covering $-0.35-+0.35$ in pseudorapidity and azimuthal angle of $180^{\circ}$ in total. The central arm consists of tracking detectors, particle identification detectors and electromagnetic calorimeters (EMCal) at the end. Charged particles and photons can be detected by these arms. The high energy photon trigger by means of the EMCal is implemented and it is utilized to collect high transverse momentum $\left(p_{T}\right)$ photons, $\pi^{0} \mathrm{~s}$ and jets. The other two spectrometers are composed of three stations of tracking chambers and a 5-layer sandwich of chambers and a steel to identify muons (muon arm).

\section{RECENT RESULTS}

In this conference, many talks from PHENIX are presented. The latest results from the 2005 run include single transverse-spin asymmetry of charged pions [4] and double helicity asymmetry of $\pi^{0}[5]$ and $J / \psi[6]$. The study of helicity correlated difference in jet intrinsic $k_{T}$ to study the orbital angular momentum of the parton is presented[7]. Many other works are ongoing and some of them are introduced in this report.
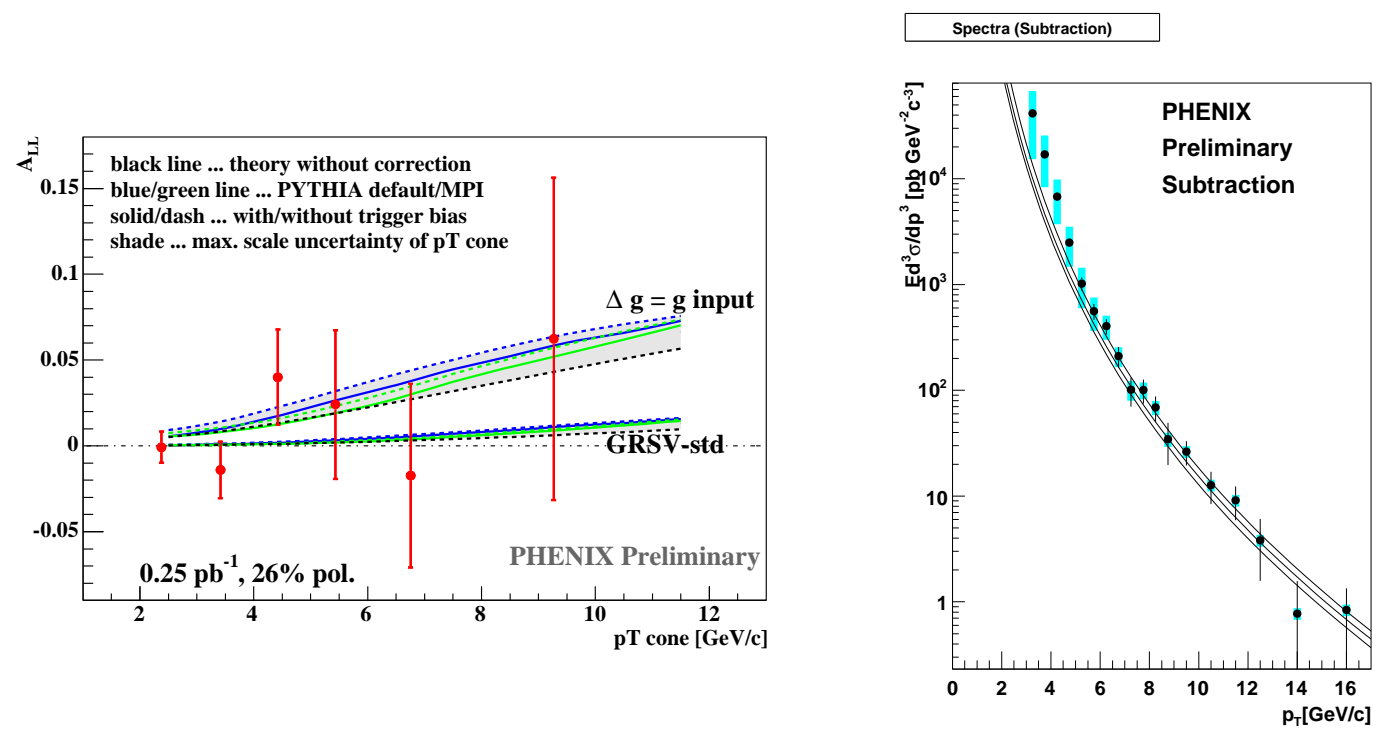

FIGURE 1. (a)-left; $A_{L L}$ of the jet as a function of the observed $p_{T}$ ( $p_{T}$ cone). The theoretical curves are normalized based on the PYTHIA simulation. Black; without scaling (original), Blue; scaled using default PYTHIA, Green; scaled using PYTHIA with the correction of multi-parton interaction which reproduces the data better than default PYTHIA. The bias of high energy photon trigger is (not) considered in solid (dashed) line. (b)-right; The cross section of prompt photons. The black bars and blue bands denote the statistical and systematic uncertainty respectively. The line is the NLO pQCD calculation with scale of $p_{T} / 2$ (upper curve), $p_{T}$ and $2 p_{T}$ (lower curve).

One of the important channels is the prompt photon to study $\Delta g$. While the $\pi^{0} A_{L L}$ with high statistics strongly constrains the size of $\Delta g$, it is difficult to determine the sign of the $\Delta g$. The $\pi^{0} A_{L L}$ can be roughly described as the quadratic equation of $\Delta g$ since $\pi^{0}$ production with high $p_{T}$ originates in $g g, g q$ and $q q$ scatterings, where $g(q)$ denotes gluon (quark). This ambiguity can be resolved by measuring $A_{L L}$ for the prompt photon production which is dominated by $g q$ scattering. Figure 1 (b) shows the cross section of the prompt photon measured by the central arms in the 2003 run with the theoretical calculation based on next leading order (NLO) perturbative quantum chromodynamics 
(pQDC). The theory describes the data well at the $p_{T}>5 \mathrm{GeV}$. Because of the low statistics, it is necessary to accumulate more data with high luminosity and polarization to obtain precision results of the asymmetry.

Another effective probe to determine $\Delta g$ is the $A_{L L}$ measurement of inclusive jet production. In addition to high statistics, the advantage over the $\pi^{0}$ is that jet is free from the uncertainty of the fragmentation. In the analysis, the jet is measured by collecting the photons and charged particles within the cone of 0.3 in the central region. The fraction of the jet momentum observed by the detector to the initial momentum of the scattered parton is evaluated by the simulation to be $\sim 80 \%$. Figure 1 (a) shows the results of the jet $A_{L L}$ in the 2003 run. Two theoretical curves are also shown, one using the best global fit to the inclusive DIS data (GRSV-std) and another one using a polarized gluon distribution equal to the unpolarized distribution $(\Delta g=g)$ [8]. The horizontal axis is the jet $p_{T}$ observed by the PHENIX detector $\left(p_{T}\right.$ cone). The theoretical curves are scaled along horizontal axis based on the PYTHIA simulation. The precision of the data will be much improved by the data taken in the 2005 run.

\section{FOR THE FUTURE}

For the future program, it is planned to extract polarized anti-quark distribution through the $W$ bosons, whose coupling is flavor sensitive and quarks and anti-quarks can be separated. It is necessary to operate RHIC with higher $\sqrt{s}$ to accumulate enough statistics of $W$. In the 2005 run, RHIC tested the operation at $\sqrt{s}=410 \mathrm{GeV}$ and we confirmed that the beam is accelerated with significant polarization by measuring single transverse-spin asymmetry for the forward neutron production.

New hardware is being developed for the future plan. One of them is the silicon vertex tracker to make the acceptance of jet wider and tag the heavy flavor particle by detecting the displaced vertex. The trigger upgrade for the muon arms is in progress for the $W$ detection by tagging high momentum muons.

\section{SUMMARY}

PHENIX collected the data in the first long polarized proton run in 2005. The measurement of the double helicity asymmetry in $\pi^{0}$ production constrains $\Delta g$. It is expected that the study of other channel will help the systematic understanding about $\Delta g$. Many other efforts, including the measurement of $A_{N}$, jet $k_{T}$, spin transfer of $\Lambda$ and $\mathrm{J} / \psi$ polarization, are underway. The commissioning of the polarized proton-proton collision at $\sqrt{s}=410$ $\mathrm{GeV}$ was successfully done in the 2005 run. As well as the accelerator development, the detector upgrades in the PHENIX is ongoing for the future programs.

\section{REFERENCES}

1. K. Adcox et al. (PHENIX Collaboration), Nucl. Inst. Meth. A499 (2003) 469.

2. M. Hirai, S. Kumano and N. Saito, Phys. Rev. D69 (2004) 054021.

3. S. S. Adler et al. (PHENIX Collaboration), Phys. Rev. Lett. 93 (2004) 202002.

4. K. Eyser et al. (PHENIX Collaboration), These proceedings.

5. K. Boyle et al. (PHENIX Collaboration), These proceedings.

6. M. Liu et al. (PHENIX Collaboration), These proceedings.

7. R. Hobbs et al. (PHENIX Collaboration), These proceedings.

8. B. Jäger, M Stratmann and W. Vogelsang, Phys. Rev. D70 (2004) 034010. 